\title{
PRODUÇÃO DE MORANGUEIRO DA CV. CAMINO REAL EM SISTEMA DE TÚNEL ${ }^{1}$
}

\author{
ROBERTO PEDROSO DE OLIVEIRA ${ }^{2}$, WALKYRIABUENO SCIVITTARO ${ }^{3}$, DAIANAFINKENAUER ${ }^{4}$
}

RESUMO - O objetivo deste trabalho foi avaliar a produção da cultivar de morangueiro Camino Real, recentemente introduzida da Califórnia, comparando-a com a das cultivares Aromas e Camarosa, nas condições climáticas do Rio Grande do Sul. O experimento foi realizado em Pelotas, utilizando-se do sistema de produção sob túnel e mudas importadas do Chile. A irrigação foi feita por gotejamento, e a adubação, fornecida via água de irrigação. Em maio de 2006, foi feito o transplantio das mudas no espaçamento de $35 \mathrm{~cm}$ entre linhas e entre plantas. Semanalmente, de agosto a dezembro, foram analisadas as variáveis massa fresca e número médio de frutos produzidos. O delineamento experimental foi de blocos casualizados com parcelas subdivididas no tempo, com quatro repetições. As unidades experimentais foram constituídas por 21 plantas. Verificou-se que a produção da cultivar Camino Real (1.121,2 g de frutos comerciais por planta por ano) é semelhante à da 'Aromas' (1.043,3 g) e à da 'Camarosa' (1.038,3 g). Os frutos da 'Camino Real' são maiores e de maior massa (24,6 g) em relação aos da 'Camarosa' (19,5 g) e da 'Aromas' (17,9 g), sendo, no entanto, produzidos em menor número. A máxima massa fresca obtida da cv. Camino Real é de 30,7 g, na $12^{\mathrm{a}}$ semana de colheita.

Termos para indexação: Fragaria $\mathrm{x}$ ananassa Duch., 'Aromas', 'Camarosa', produtividade, massa fresca dos frutos.

\section{CAMINO REAL STRAWBERRY YIELD IN TUNNEL SYSTEM}

\begin{abstract}
The aim of this work was to evaluate the 'Camino Real' strawberry yield that is a cultivar recently introduced from California, and to compare it to 'Aromas' and 'Camarosa' under climatic conditions of the state of Rio Grande do Sul. The experiment was carried out in Pelotas, using tunnel system, fertirrigation and runners imported from Chile.. The runners were transplanted to space of $35 \mathrm{~cm}$ between rows and between plants, in May 2006. Weekly, from August to December, data on fruit fresh matter and number of fruits produced in each experimental unit were collected. The experimental design was a randomized complete block with split plot, with four replications. The experimental unit consisted of 21 plants. The fruit yield of 'Camino Real' (1121.2 g of commercial fruits per plant per year) is similar to Aromas (1043.3 g) and to Camarosa $(1038.3 \mathrm{~g})$ cultivars. Their fruits are bigger and with higher fresh matter (24.6 g) than Camarosa (19.5 g) and Aromas (17.9 g) cultivars, but in small number. The highest average of fresh fruit matter of the Camino Real cultivar is $30,7 \mathrm{~g}$, in the $12^{\text {th }}$ week of harvest.
\end{abstract}

Index terms: Fragaria x ananassa Duch., 'Aromas', 'Camarosa', yield, fruit fresh matter.

\section{INTRODUÇÃO}

O morangueiro (Fragaria $\mathrm{x}$ ananassa Duch.) pertence à família das Rosáceas, sendo um híbrido resultante das espécies americanas $F$. chiloensis, $F$. virginiana e $F$. ovalis, e da européia Fragaria vesca (Ronque, 1998). No Brasil, a cultura encontra-se difundida em regiões de clima temperado e subtropical, onde se produz morango para consumo in natura e para a industrialização (Santos, 2003). A produção mundial de morango é de 3,1 milhões de toneladas por ano, e a brasileira, de 37,6 mil toneladas, sendo esta última obtida em uma área estimada de 3,5 mil hectares, com destaque para os Estados de Minas Gerais (41,4\%), Rio Grande do Sul (25,6\%) e São Paulo (15,4\%) (IEA, 2007).

As cultivares de morangueiro mais utilizadas na região Sul do Brasil provêm dos Estados Unidos, destacando-se a 'Aromas', 'Camarosa', 'Diamante', 'Oso Grande' e 'Ventana', da Universidade da Califórnia, e 'Dover' e 'Sweet Charlie', da Universidade da Flórida (Oliveira et al., 2005). No Rio Grande do
Sul, 'Camarosa' e 'Aromas' são, respectivamente, as cultivares de dias curtos e de dias neutros mais utilizadas. 'Camarosa' foi lançada em 1993, tendo plantas vigorosas e com folhas grandes de coloração verde-escura. O ciclo é precoce, com alta capacidade de produção, quando comparada à Aromas e Diamante. Os frutos são grandes, uniformes, de coloração vermelho-escura, polpa firme e sabor subácido, sendo indicados tanto para consumo in natura quanto para industrialização (Santos, 2003). Já a cultivar Aromas foi lançada em 1997, sendo muito produtiva, com hábito de crescimento ereto, frutos grandes, coloração vermelha acentuada, sabor agradável e qualidade excelente para consumo in natura e industrialização (Shasta Nursery, 2007).

Em razão da diversidade edafoclimática existente no País, o pequeno número de cultivares disponível tem sido um dos principais obstáculos ao desenvolvimento da cultura do morangueiro, tanto nas regiões ainda não produtivas, quanto naquelas em que já está sendo produzido. Por isso, é importante incentivar programas nacionais de melhoramento genético e de introdução de cultivares geradas em outros países.

A cultivar Camino Real é uma das novidades no mercado

1(Trabalho 124-07). Recebido em: 15-05-2007. Aceito para publicação em: 16-04-2008.

${ }^{2}$ Eng. Agr., Doutor, Pesquisador da Embrapa Clima Temperado, C. Postal 403, 96001-970 Pelotas-RS. Bolsista do CNPq. Email: rpedroso@cpact.embrapa.br ${ }^{3}$ Eng. Agr., Doutor, Pesquisador da Embrapa Clima Temperado. Email: wbscivit@cpact.embrapa.br

${ }^{4}$ Graduanda de Biologia. Universidade Católica de Pelotas, Felix da Cunha, 412, 96010-000 Pelotas-RS 
brasileiro, tendo sido desenvolvida na Universidade da Califórnia, em 2001, e, recentemente, introduzida no Brasil. Segundo Shaw \& Larson (2007), essa cultivar apresenta alta capacidade de produção. As plantas são relativamente pequenas, compactas e eretas. Os frutos são de sabor agradável, grandes, firmes, com epiderme e polpa vermelho-escuras, sendo recomendados para o mercado in natura e industrialização. Essa cultivar é suscetível ao oídio, relativamente resistente à antracnose e resistente à verticilose $\mathrm{e}$ às podridões do colo e do rizoma.

O objetivo deste trabalho foi avaliar a produção da cultivar de morangueiro Camino Real, comparando-a com a das cvs. Aromas e Camarosa, nas condições climáticas do Rio Grande do Sul.

\section{MATERIAL E MÉTODOS}

O experimento foi realizado no município de Pelotas-RS (latitude 31 $46^{\prime} 00^{\prime}$, altitude de $245 \mathrm{~m}$ e temperatura média anual de $17,6^{\circ} \mathrm{C}$ ), utilizando-se do sistema de produção sob túneis. Estes apresentavam $0,5 \mathrm{~m}$ de altura na parte central, sendo sustentados por arcos de arame galvanizado número 6 e cobertos com filme de polietileno transparente aditivado, com 100 micras de espessura.

O ensaio foi realizado em canteiros com 1,2 $\mathrm{m}$ de largura e $0,15 \mathrm{~m}$ de altura, espaçados entre si em $0,8 \mathrm{~m}$, constituído por solo previamente corrigido quanto à acidez, $\mathrm{pH}$ 6,0 (Sociedade, 2004). Utilizou-se um sistema de irrigação por gotejamento, sendo as mangueiras distribuídas nos canteiros anteriormente ao seu revestimento com filme de polietileno preto, com 30 micras de espessura. As mudas de morangueiro foram transplantadas em 18 de maio de 2006, sendo dispostas três linhas por canteiro. O espaçamento entre linhas e entre plantas foi de $0,35 \mathrm{~m}$.

Avaliaram-se três cultivares de morangueiro (Aromas, Camarosa e Camino Real), usando mudas procedentes do Chile. Utilizou-se um delineamento experimental de blocos ao acaso, com parcelas subdivididas no tempo, e quatro repetições, sendo as unidades experimentais constituídas por 21 plantas.

Durante o experimento, o controle das plantas daninhas e a remoção de folhas secas ou com sintomas de doenças, de estolões e de frutos estragados ou com defeitos foram feitos manualmente. A irrigação das plantas foi realizada diariamente, de acordo a necessidade hídrica. Semanalmente, estas foram fertirrigadas, com aplicações alternadas dos fertilizantes $\mathrm{KSC}^{\circledR}$, $\mathrm{KSC}^{\circledR}$ e nitrato de cálcio (500 g por 1.000 plantas). O tratamento fitossanitário incluiu duas aplicações de iprodione e uma de azoxystrobin, para o controle de fungos, uma aplicação de abamectina, para o controle de ácaros, e a utilização de iscas, para controle da broca-dos- frutos (Lobiopa insularis) e de camundongos.

O manejo dos túneis plásticos consistiu em sua abertura do lado oposto aos ventos predominantes, até a altura de $0,4 \mathrm{~m}$ nas manhãs mais quentes, sendo fechados nos finais de tarde e antes da ocorrência de chuvas e/ou neblinas.

Semanalmente, determinaram-se a massa fresca e o número de frutos produzidas por unidade experimental. A massa fresca dos frutos foi calculada pela razão entre essas duas variáveis. Determinou-se, ainda, a produção acumulada de frutos ao longo do período de colheita. Para o experimento, consideraram-se, apenas, os frutos comercializáveis, sendo descartados aqueles com defeitos de formato, podres ou com massa inferior a $3 \mathrm{~g}$ (CEAGESP, 2002). O período de colheita dos frutos estendeu-se de agosto a dezembro de 2006, totalizando 20 avaliações semanais. Porém, para a análise estatística, agruparamse os dados a cada quatro semanas, perfazendo cinco períodos de avaliação. Os dados foram submetidos à análise de variância, comparando-se as médias do fator cultivar pelo teste de Tukey $(\mathrm{p}<0,05)$ e do fator período de avaliação, por análise de regressão polinomial.

\section{RESULTADOS E DISCUSSÃO}

A cultivar Camino Real mostrou-se tão produtiva quanto a 'Camarosa' e a 'Aromas', sendo colhidas, respectivamente, $1.121,2 ; 1.038,3 \mathrm{e} 1.043,3 \mathrm{~g}$ de frutos comerciais por planta (Tabela 1). Larson \& Shaw (2007), que participaram do desenvolvimento dessas cultivares, já haviam relatado elevada produtividade, com leve tendência de superioridade da 'Camino Real'. Este resultado também demonstra a grande adaptabilidade das três cultivares obtidas na Califórnia às condições climáticas do Rio Grande do Sul. Ainda em relação às produtividades obtidas, estas foram muito satisfatórias, tendo sido bastante superiores aos $300 \mathrm{~g}$ por planta considerado na prática, como patamar de viabilidade econômica para a cultura do morangueiro (Rebelo \& Balardin, 1997), aos 300-400 g que é a média do Rio Grande do Sul (Pagot \& Hoffmann, 2003) e aos $700 \mathrm{~g}$ por planta utilizado como referencial de produtividade adequada pelo Agrianual (2007). Na mesma localidade e condições de cultivo do presente experimento, Oliveira et al. (2006) obtiveram, em média, 740 g de frutos por planta da cv. Camarosa e $709 \mathrm{~g}$ da 'Aromas', na safra de 2005, diferença que pode ser explicada pelo morangueiro ser altamente influenciado pelas condições de temperatura e de fotoperíodo, vigentes durante o período de desenvolvimento das plantas e de produção das frutos (Ronque, 1998). Ainda em relação às cultivares Camarosa e Aromas, na Itália, sob condições de ambiente protegido, D'Anna \& Prinzivalli (2002) obtiveram índices de produtividade bastante inferiores, respectivamente de $428 \mathrm{~g}$ e de $382 \mathrm{~g}$ de frutos por planta, e Leis et al. (2002b), de 920 g por planta da cv. Camarosa. No Brasil, no sul de Minas Gerais, Duarte Filho et al. (2006) obtiveram 484 g por planta da cultivar Camarosa. Para a cultivar Camino Real, os resultados apresentados referem-se às primeiras quantificações de produtividade realizadas no País, praticamente não se dispondo de relatos externos aos Estados Unidos.

De uma forma geral, as três cultivares apresentaram alta regularidade de produção, o que é uma característica muito valorizada pelos produtores (Rebelo \& Balardin, 1997), não encontrada em algumas cultivares, como a Ventana, cujas floradas são intensas e periódicas (Oliveira et al., 2006).

Ao longo do período de colheita, o número de frutos produzidos por planta da 'Camino Real' $(44,9)$ foi inferior à da 'Camarosa' $(55,0)$ e da 'Aromas' $(55,2)$, que não diferiram entre si. No entanto, a 'Camino Real' apresentou maior massa média de 
frutos (24,6 g), seguida por 'Camarosa' (19,5 g) e 'Aromas' (17,9 g) (Tabela 1). Na Itália, D’Anna \& Prinzivalli (2002) obtiveram média de 15,2 g para 'Camarosa' e de 16,3 g para 'Aromas', enquanto Leis et al. (2002b) obtiveram 23,5 g para 'Camarosa'. Quanto à cv. Camino Real, o valor obtido em Pelotas (24,6 g) foi bastante próximo aos obtidos por Chandler et al. (2005), na Flórida (24 g), e por Shaw \& Larson (2007), na Califórnia (27,2 g). Chandler et al. (2005) relatam que uma das principais vantagens da cv. Camino Real se refere à baixa porcentagem de produção de frutos pequenos (menos de $1 \% \mathrm{com}$ massa inferior a $10 \mathrm{~g}$ ). Isto provavelmente ocorre em função de a polinização ocorrer satisfatoriamente em uma faixa maior de temperatura (Shaw \& Larson, 2007) e da produção de apenas um fruto por inflorescência (Shimizu, 2005).

Para as três cultivares de morangueiro estudadas, o efeito do período de avaliação sobre a produção de frutos ajustou-se a modelos quadráticos de regressão. Apenas a 'Camarosa' atingiu o valor máximo de produção dentro do período de avaliação, o qual correspondeu à $15^{\mathrm{a}}$ semana de colheita. Nas primeiras doze semanas de colheita, correspondentes aos meses de agosto, setembro e outubro, a maior produção de frutos por planta foi determinada para a cultivar Camarosa' em relação à 'Camino Real' e 'Aromas', ocorrendo o inverso nas últimas quatro semanas, correspondentes ao mês de dezembro (Figura 1). A precocidade de produção da 'Camarosa' em relação à 'Aromas' já havia sido relatada por Oliveira et al. (2006) e à 'Camino Real' por Larson \& Shaw (2007). Segundo Santos (2003), a 'Camarosa' encontra-se no grupo das cultivares de morangueiro de maior precocidade de produção. Ainda na Figura 1, verifica-se o comportamento diferenciado da cv. Aromas, única de dias neutros dentre as estudadas, com produção crescente ao longo do período de colheita, inclusive após a $20^{\mathrm{a}}$ semana de colheita.

Também a variação na massa fresca de frutos ao longo do período de colheita, para todas as cultivares avaliadas, ajustouse ao modelo quadrático (Figura 2). A maior massa fresca de fruto foi obtida na $12^{\text {a }}$ semana de colheita para a 'Camino Real' $(30,7 \mathrm{~g})$, na $14^{\mathrm{a}}$ semana para a 'Aromas' $(21,0 \mathrm{~g})$ e na $11^{\mathrm{a}}$ semana para a 'Camarosa' (24,0 g). Esses resultados evidenciam o potencial da cultivar Camino Real para a produção de frutos de tamanho extra (>14 g), segundo classificação proposta por Rebelo \& Balardin (1997). Na Figura 2, observa-se, também, que os frutos da cultivar Camino Real apresentaram maior massa média durante todo o período de colheita avaliado. Os frutos da 'Aromas' foram os de menor massa fresca até a $16^{\mathrm{a}}$ semana de colheita, quando se tornaram maiores do que os da 'Camarosa'. Esses resultados contradizem o comportamento normalmente relatado das cultivares de morangueiro, que apresentam maior massa fresca no início da colheita (Leis et al., 2002a). Porém, isso depende muito da época em que é feito o transplantio das mudas e das condições ambientais durante seu cultivo (Santos, 2003).
TABELA 1 - Efeito da cultivar na produção acumulada, número e massa fresca de frutos de morangueiro (Fragaria $\mathrm{x}$ ananassa Duch.) colhidas de agosto a dezembro na região de Pelotas-RS.

\begin{tabular}{lccc}
\hline Cultivar & $\begin{array}{c}\text { Produção total } \\
\left(\mathrm{g} \mathrm{planta}^{-1}\right)\end{array}$ & $\begin{array}{c}\text { Número de frutos } \\
\left(\text { frutos planta }^{-1}\right)\end{array}$ & $\begin{array}{c}\text { Massa fresca } \\
\left(\mathrm{g} \mathrm{fruto}^{-1}\right)\end{array}$ \\
\hline Camino Real & $1.121,2 \mathrm{a}$ & $44,9 \mathrm{~b}$ & $24,6 \mathrm{a}$ \\
Camarosa & $1.038,3 \mathrm{a}$ & $55,0 \mathrm{a}$ & $19,5 \mathrm{~b}$ \\
Aromas & $1.043,3 \mathrm{a}$ & $55,2 \mathrm{a}$ & $17,9 \mathrm{c}$ \\
\hline CV $(\%)$ & 11,5 & 11,5 & 3,0 \\
\hline
\end{tabular}

Médias seguidas de mesma letra, nas colunas, não diferem significativamente entre si, pelo teste de Tukey $(5 \%)$.

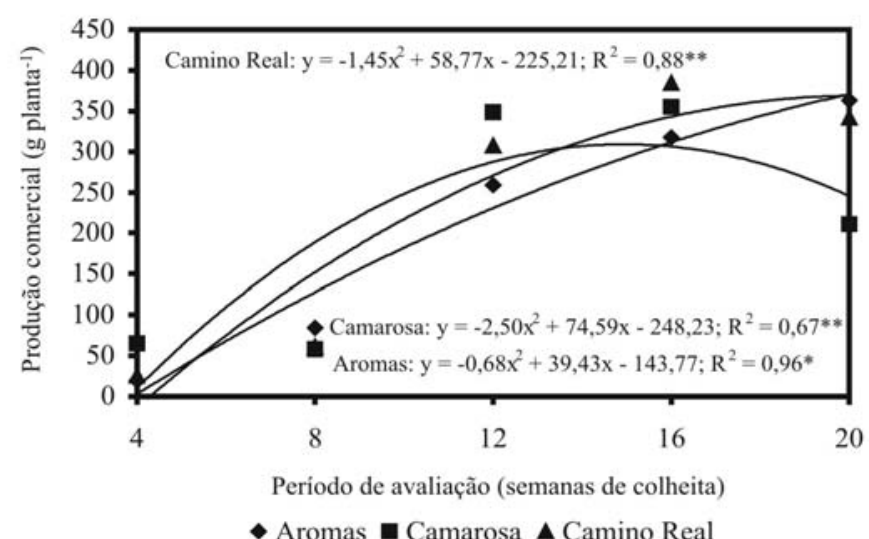

FIGURA 1 - Evolução da produção comercial de três cultivares de morangueiro (Fragaria $\mathrm{x}$ ananassa Duch.), ao longo do período de colheita.

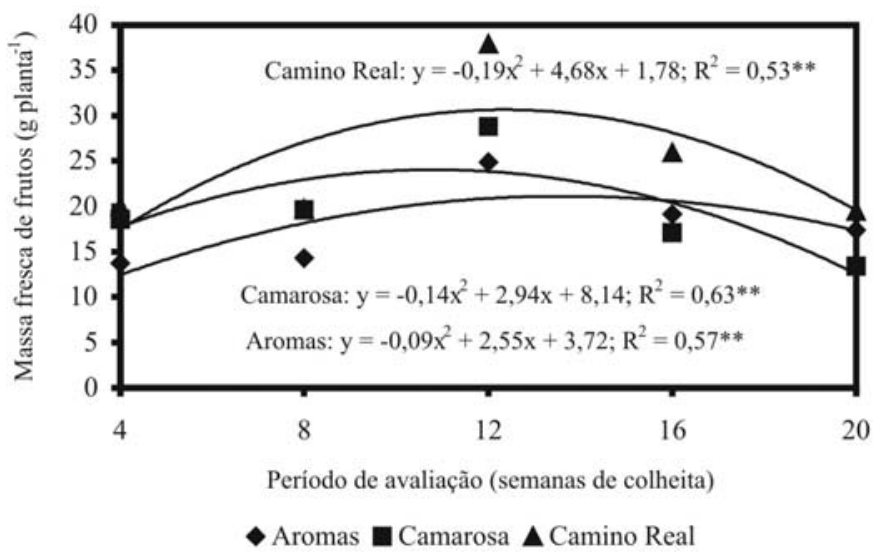

FIGURA 2 - Evolução da massa fresca de três cultivares de morangueiro (Fragaria x ananassa Duch.), ao longo do período de colheita. 


\section{CONCLUSÕES}

1- A produção de frutos de morango da cultivar Camino Real é similar à das cultivares Aromas e Camarosa.

2- A produção de frutos da 'Camino Real' é mais tardia do que a da 'Camarosa'.

3- Os frutos da cv. Camino Real são maiores e com maior massa fresca do que os da 'Camarosa' e da 'Aromas', porém são produzidos em menor número.

\section{AGRADECIMENTOS}

À FAPERGS e ao CNPq, pelo apoio financeiro ao projeto e concessão de bolsas de estudo.

\section{REFERÊNCIAS}

AGRIANUAL 2007: anuário da agricultura brasileiraSão Paulo: FNP Consultoria e AgroInformativos, 2006. p.424-427.

CEAGESP. Normas de classificação do morango. São Paulo: CEAGESP/ Centro de Qualidade em Horticultura, 2002. 8p. (Documentos, 22)

CHANDLER, C.K.; SUMLER JR., J.C.; RONDON, S. Evaluation of strawberry cultivars grown under a high plastic tunnel in west central Florida. Proceedings of the Florida State Horticultural Society, Winter Haven, v.118, p.113-114, 2005.

D'ANNA, F.; PRINZIVALLI, C. Due anni di studio su varietà di fragola per l'ambiente protetto meridionale. Rivista di Frutticoltura e di Ortofloricoltura, Bologna, v.64, n.6, p.61-68, 2002.

DUARTE FILHO, J.; GAMBARDELLA, M.; ANTUNES, L.E.C.; DIAS, J.P.T.; PÁDUA, J.G. Desempenho agronômico de diferentes cultivares de morangueiro a partir de mudas frescas originadas do Chile. In: SIMPÓSIO NACIONAL DO MORANGO, 3., 2006, Pelotas. Resumos expandidos ... Pelotas: Embrapa Clima Temperado, 2006. p.127-133.

IEA - INSTITUTO DE ECONOMIA AGRÍCOLA. Pólos de produção do morango. Disponível em: $<\mathrm{http}$ ://www.iea.sp.gov.br/ out/vertexto.php?codtexto=11>. Acesso em: 11 jan. 2007.

LARSON, K.; SHAW, D. The Ventana strawberry cultivar. Disponível em: <http://fruitsandnuts.ucdavis.edu/strawberry/ Website_Ventana_Strawberry_Cultivar_final.pdf $>$. Acesso em: 5 maio 2007 .

LEIS, M.; CASTAGNOLI, G.; MARTINELLI, A. Ariel. Rivista di Frutticoltura e di Ortofloricoltura, Bologna, v.64, n.6, p.47, 2002a.
LEIS, M.; CASTAGNOLI, G.; MARTINELLI, A. Naiad Civl35. Rivista di Frutticoltura e di Ortofloricoltura, Bologna, v.64, n.6, p.48-49, 2002b.

OLIVEIRA, R.P.; NINO, A.F.P.; SCIVITTARO, W.B. Mudas certificadas de morangueiro: maior produção e melhor qualidade da fruta. A Lavoura, Rio de Janeiro, v.108, p.35-38, 2005.

OLIVEIRA, R.P.; SOUZA, T.M.; SCIVITTARO, W.B. Ventana: nova cultivar de morangueiro recomendada para o Rio Grande do Sul. Pelotas: Embrapa Clima Temperado, 2006. 4p. (Comunicado Técnico, 138).

PAGOT, E.; HOFFMANN, A. Produção de pequenas frutas. In: SEMINÁRIO BRASILEIRO SOBRE PEQUENAS FRUTAS, 1 ., 2003, Vacaria. Anais ... Bento Gonçalves: Embrapa Uva e Vinho, 2003. p.9-17. (Documentos, 37).

REBELO, J.A.; BALARDIN, R.S. A cultura do morangueiro. 3.ed. Florianópolis: EPAGRI, 1997. 44p. (Boletim Técnico, 46).

RONQUE, E.R.V.Cultura do morangueiro; revisão e prática. Curitiba: Emater, 1998.206p.

SANTOS, A.M. Cultivares. In: SANTOS, A.M.; MEDEIROS, A.R.M. (Ed.). Morango: produção. Brasília: Embrapa Informação Tecnológica, 2003. p.24-30. (Frutas do Brasil, 40).

SHASTA NURSERY. Aromas-CN209. Disponível em: $<$ http:// www.rootstock.com/variety.html\#anchor70036>. Acesso em: 5 maio 2007.

SHAW, D.; LARSON, K. The Camino Real strawberry cultivar. Disponível em: <http://fruitsandnuts.ucdavis.edu/strawberry/ Website_Camino_Real_description_final2.pdf $>$. Acesso em: 5 maio 2007.

SHIMIZU, H.K. Novas cultivares de morangueiro em 2005. Bioagro, Araucária, v.1, p.6, 2005.

SOCIEDADE BRASILEIRA DE CIÊNCIA DO SOLO. Manual de adubação e calagem para os Estados do Rio Grande do Sul e de Santa Catarina. 10.ed. Porto Alegre: Comissão de Química e Fertilidade do Solo, 2004. 400 p 